\title{
PROJECT PLANNING USING AN INTERACTIVE, STRUCTURED MODELING ENVIRONMENT
}

\author{
Ian Flood \\ Rinker School, College of Design, Construction, and Planning \\ University of Florida \\ Gainesville, FL. 32611, U.S.A.
}

\begin{abstract}
Planning construction projects typically makes use of the activity network based Critical Path Method (CPM), since it is very simple to use and reasonably versatile. Most other planning techniques are either aimed at specialized types of construction work (such as linear scheduling techniques) or are peripheral tools to be used in conjunction with these tools (such as n-D CAD). Discrete-event simulation has also been used for construction planning, and while it is extremely versatile, it lacks the simplicity in use of CPM and so has not been widely adopted within the industry. This paper goes back to first principles, identifying the needs of construction project planning and how existing tools meet (or fail to meet) these requirements. Based on this, it proposes a new modeling paradigm better suited to contemporary construction project planning. The principles of the method are demonstrated with a range of examples from construction.
\end{abstract}

\section{INTRODUCTION}

The last 100 years have seen the development of a wealth of tools for project planning. An analysis of the genealogy of these tools (Flood et al., 2006) indicates that they fall into three main categories (the Critical Path Methods (CPM), linear scheduling techniques, and simulation) and most other tools are either enhancements or an integration of these techniques. Arguably, 4D-CAD and nDCAD (see, for example, Koo \& Fischer 2000, and Issa et al 2003) can be deemed a fourth category of planning tool, but only if the planning variables (schedule, costs, etc.) are rendered on to the 3D or 4D image of the facility; systems that simply link a 3D or 4D image of the facility to a regular schedule, can only be considered an enhancement of existing methods.

Unfortunately, each category of planning tool is only applicable to a certain class of construction planning problem. CPM is best suited to planning projects at a fairly general level of detail and that have little or no repetition of tasks. Linear scheduling (see, for example, Matilla and Abraham 1998) is targeted at projects where there is repetition at a high level (such as tunnel, highway, or high-rise construction), but cannot handle nonrepetitive work and, moreover, can only consider a single sequence of tasks where each task has its own crew. While simulation (see, for example, Halpin \& Woodhead, 1976, Sawhney et al., 1998, and Hajjar \& AbouRizk, 2002) can in principle be applied to any type of construction project, the work involved in defining and validating a model means that in practical terms it is best suited to systems comprising repetitive tasks.

It has long been noted that CPM is not very good at modeling construction work that is repetitive in nature (such as tunneling or high-rise construction) (Harris \& Ioannou, 1998). When applied to repetitive work, CPM models are unduly complicated and provide little understanding of the interactions between repetitive construction tasks. On the other hand, while simulation is very versatile at representing repetitive work, it is unnecessarily complicated and not very insightful when it comes to modeling non-repetitive work. Linear scheduling methods, in comparison, are very easy to understand and provide great insight into the behavior of a construction system, but they cannot be used at all to model non-repetitive work and include some simplistic assumptions which often make it difficult to model real-world repetitive work. Velocity diagrams (a linear scheduling technique), for example, cannot easily represent operations that use flexible crews, that is, crews that may be split-up occasionally to work temporarily on several tasks and then regrouped later (which is often the way they are utilized in repetitive working environments).

Regrettably, there is no single tool well suited to modeling the broad spectrum of repetitive and nonrepetitive construction work in terms of versatility, insight, and ease of use. Thus, planners are left with two options: (i) to use a selection of planning tools or; (ii) to use a single tool for planning all types of work even though it will not always be the most appropriate. The first choice is rarely adopted since it requires the planner, and all other involved parties, to be proficient in the use of several software packages some of which they may only use on rare occasions; moreover, the results from the 
different tools cannot be readily integrated into a single analysis. Most often, a CPM approach is adopted and applied to all situations, compromising modeling of the repetitive elements of a project.

Another issue is that the principles upon which these tools are based are often flawed or biased towards a view of planning that is out dated. CPM, for example, has a time-centric view of planning, and treats other parameters and constraints very much as secondary issues. For example, distance buffers between concurrent linear tasks must be converted into a time equivalent, which is both misleading and un-insightful.

This paper goes back to basics and attempts to develop a new modeling paradigm that is relevant to all issues in contemporary planning and applicable to all types of construction project.

\section{STRUCTURED MODELING}

The first principle in the proposed approach to project modeling is the adoption of a strongly structured view of the work involved in a project. Structured modeling has long been recognized in systems science as a powerful way of developing and defining representations of very large and complex systems. In essence, a structured approach forms a representation of a system by decomposing it into categories of tasks and subtasks, in a top-down manner. For construction, the decomposition into tasks should be building-component oriented (as opposed to say material-type, or trade oriented) since this reflects the way in which buildings are assembled. The main advantages of a structured approach to modeling are simplified model development and revision, fewer errors in the model design, and better insight into the system being modeled (since the model provides understanding at different levels of abstraction) (AbouRizk and Hajjar (1998), Huber et al. (1990), Ceric (1994)).

The basic concept of structured modeling is already adopted in construction project planning in the form of Work Breakdown Structures (WBS's) and is even implemented in some project planning software packages. WBS's are, however, simply a classification or grouping of work tasks (to make the model more readable) and are not an integral part of the structure and operation of the model, that is, they do not help define the functionality of the model.

Consider for example, the sample project plan shown in Figure 1. The left side of the figure shows the project organized within a conventional WBS format, while the right side shows the equivalent project organized using a fully structured approach. For both approaches, each block represents a task (or sub-task) and each link represents a dependency between tasks. A fundamental difference, however, is that the proposed structured approach allows the dependencies to be defined between tasks at any level in the network (the scope of dependency of a link being all sub-tasks within the task to which it is connected) whereas the WBS approach requires all dependencies to connect between the lowest level tasks. In this example, the Tasks 1.3.1 and 1.3.2 require Tasks 1.1.2 and 1.1.3 to be completed, and Task 1.3.2 requires additionally Task 1.2 .2 to be completed. Clearly, the structured approach reduces the total number of links required to define the logic, thus making the plan easier to read and modify. Also, more subtly, the structured approach provides a better insight into the logic of the project by indicating generalized relationships (those at higher levels of abstraction). For example, it is clear from the structured format that the high-level component represented by Task 1.3 is fully dependent on the completion of the high-level component represented by Tasks 1.1, and partially dependent on completion of Task 1.2.

\section{CONSTRAINTS}

The second principle in the proposed approach to project modeling concerns its system of constraints. In any modeling system, the constraints impose restrictions on the behavior of the system, and may represent anything from limitations on the availability of resources (equipment, money, space, etc) through to a requirement for one task to maintain a minimum amount of work in advance of another task (a distance or time buffer for example). In CPM, the constraints are provided by factors such as the start and finish dependencies between the activities, the demand and availability of key resources, the periods of work, and the required durations for each activity. In linear scheduling, the constraints are the task sequence, minimum buffers between the progress of adjacent tasks, and the rate of progress of the tasks. Construction simulation, in contrast, is strongly oriented towards the use of resource availability in prescribed combinations as a means of constraining the progress of work.

Each of the above three approaches has its own advantages. The CPM approach is very simple to use, but is not well suited to projects where many of the tasks are repetitive in nature. Simulation is the most versatile allowing relatively complicated logical dependencies to be developed between tasks, but these dependencies are limited to discrete task events. The linear scheduling approach is simple to understand and allows continuous dependencies between the progress of tasks, but it lacks the versatility of the simulation approach and requires all tasks to operate along a single sequence. 

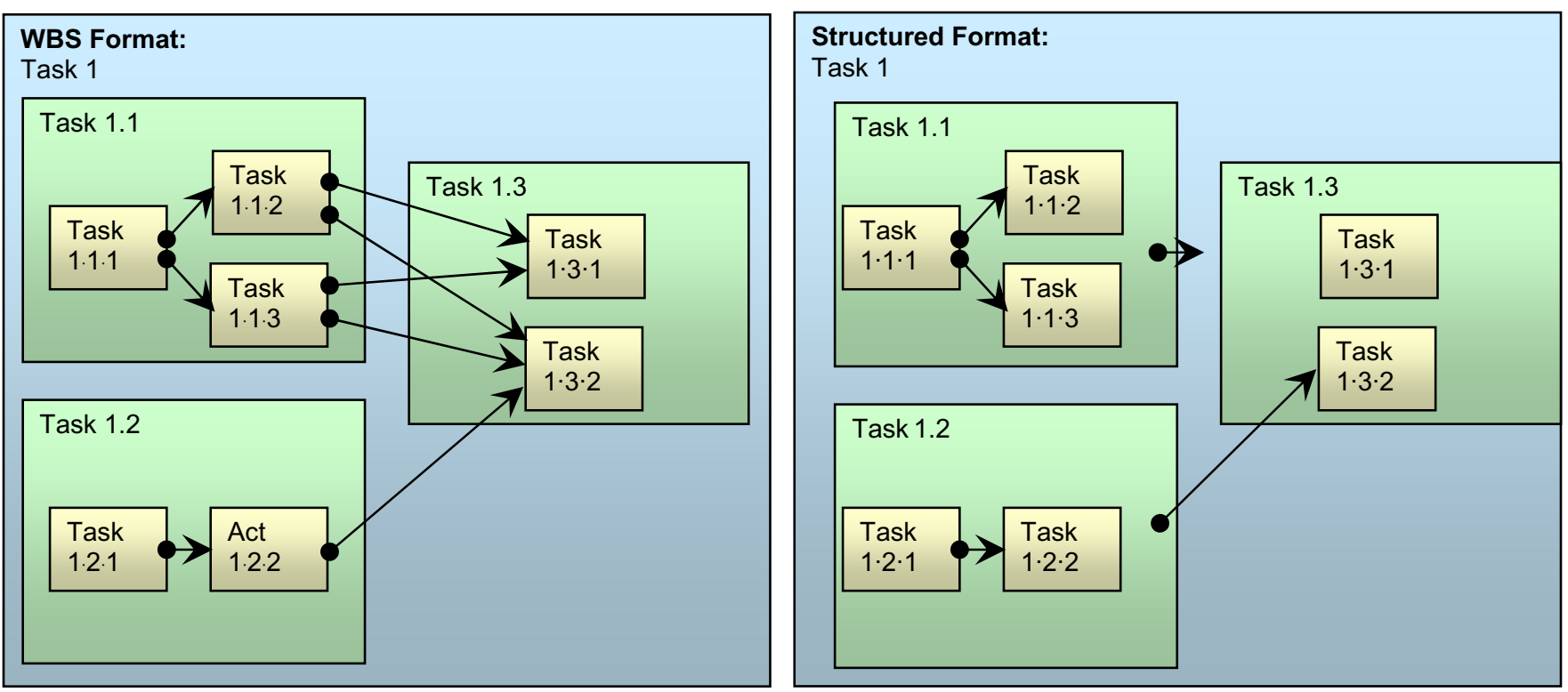

Figure 1: Comparison of the WBS and Structured Approaches to Model Representation

Ease of use and versatility in modeling (the latter of which also impacts accuracy) are necessary features for any planning tool. In the case of task constraints, this balance can be achieved using an extension of the linear scheduling approach of constrained relative progress of tasks, in combination with an extension of the CPM method of resource demands. For the proposed system, dependencies can be defined between any tasks (and at any level) to constrain their relative progress, and for any measure of work (time, distance, units completed). The advance in progress may be specified to be above or below a given value, and their may be more than one such dependency between two tasks. Thus, it may be defined that task ' $A$ ' be at least $10 \mathrm{~m}$ behind task ' $\mathrm{B}$ ', but no more than $25 \mathrm{~m}$ behind. This approach, in combination with resource availability constraints, has the versatility to model any dependency available in the CPM, linear scheduling, and simulation approaches. These points are illustrated in the following subsections.

\subsection{Representation for a Classic Simulation Problem}

Figure 2 illustrates how the proposed new approach would be used to represent a system typically analyzed using construction simulation techniques, that of concrete production and distribution utilizing a wet-concrete hopper. For comparison, the figure includes the CYCLONE diagram equivalent representation of this system (Halpin and Woodhead, 1976). The system represented comprises a $1 \mathrm{cu}-\mathrm{m}$ concrete batching plant, a $5 \mathrm{cu}-\mathrm{m}$ hopper for storing wet-concrete, and two $10 \mathrm{cu}-\mathrm{m}$ distribution trucks. In the proposed new approach (part (b) of the Figure), most of the dependencies would simply specify that preceding tasks must be completed before their successors can start. However, the link between the middle-level tasks would specify that 'Concrete Production' must be between 0 and $5 \mathrm{cu}-\mathrm{m}$ of wet concrete ahead of 'Concrete Delivery'. This would impose the logic of a $5 \mathrm{cu}-\mathrm{m}$ wetconcrete hopper between these middle-level tasks, equivalent to the central cycle of the CYCLONE model. Finally, the 'Concrete Production' task would be allocated one 'concrete mixer' resource, and each of its subtasks would require one of this resource type. Similarly, the 'Concrete Delivery' task would be allocated two 'delivery truck' resources, and each of its subtasks would require one of this resource type. These resource constraints would ensure that there was only one process thread occurring within the 'Concrete Production' task and two occurring within the 'Concrete Delivery' task.

\subsection{Representation for a Classic Linear Scheduling Problem}

Figure 3 illustrates how the proposed new approach would be used to represent a classic linear scheduling type problem. The problem represented is the construction of two underground pipelines, the first for gas and the second for water. In actual fact, this version of the problem could not be modeled using regular linear scheduling methods, since the two pipelines do not run along the same line. The water pipeline section of the project is fairly typical of a linear scheduling type problem, with a sequence of tasks that must follow each other with given distance buffers (which would be defined at the links between the tasks). 


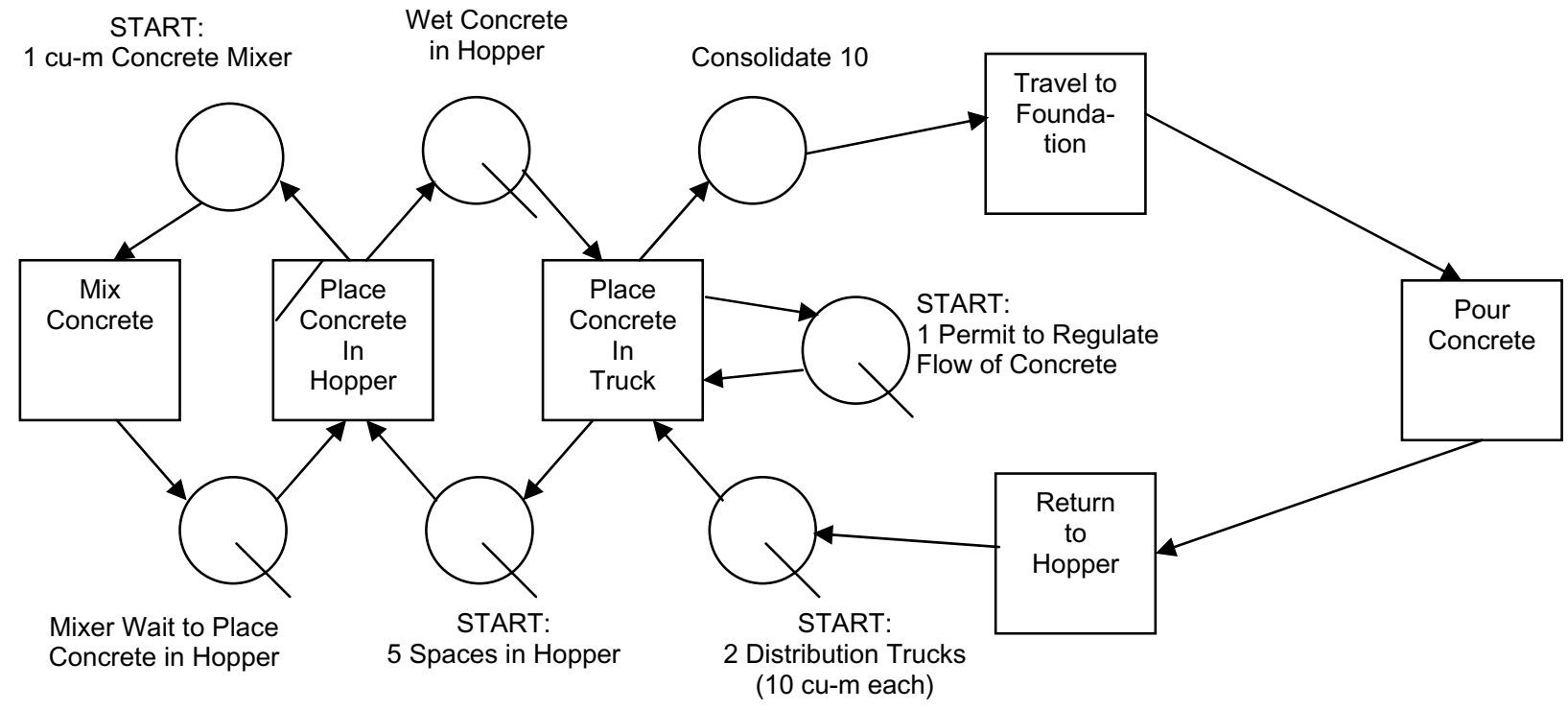

(a) CYCLONE Simulation Diagram

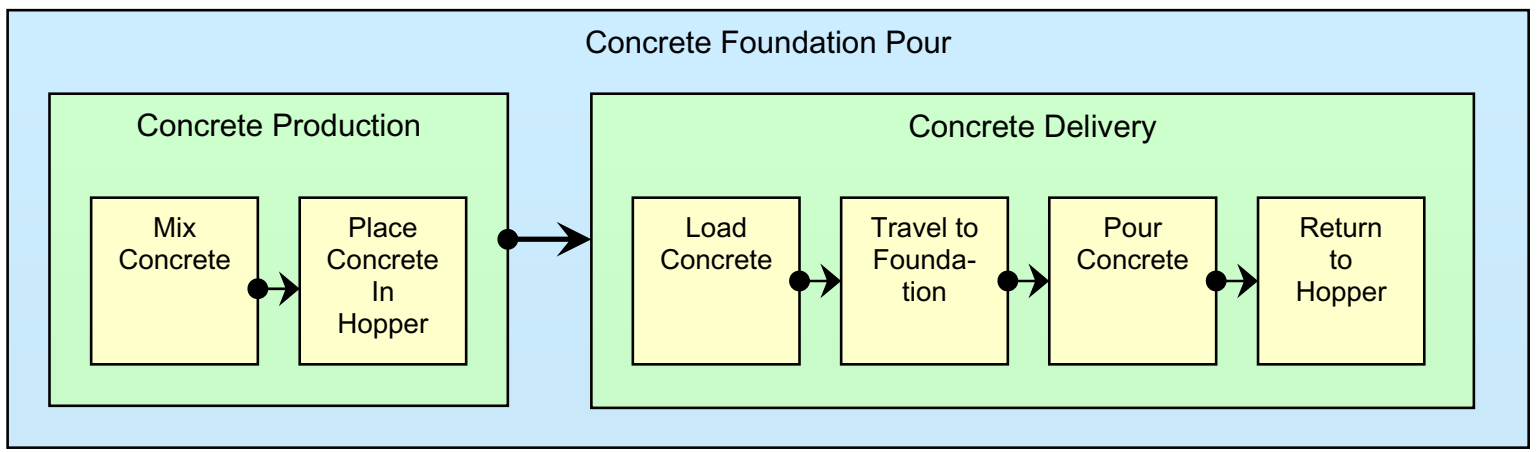

(b) Proposed New Representation

Figure 2: Concrete Foundation Pour Operation

A deviation from regular linear scheduling methods, however, is that the shoring task must be both greater than a specified minimum distance behind excavation (to avoid interference) and less than a specified maximum distance behind excavation (to minimize the chances of collapse of the trench walls). The gas pipeline includes two lay-pipe crews that operate on different sections of the pipeline, and so each is shown by its own task. The two lay-pipe crews are grouped within a higher level task.

The gas pipeline runs deeper than the water pipeline, and follows a different path. The two pipelines cross over at a given location. Since the gas pipeline is deeper than the water pipeline, it must reach and progress past the cross over point before the water pipeline. This logic is determined by the connection between the two higher level tasks: 'water pipeline' and 'gas pipeline'.

\section{VISUALIZATION}

\subsection{Structured Visualization}

Visualization of progress in a project is essential to understanding the effectiveness of a given plan, understanding the actual progress of work on site, identifying possible problems (and their ramifications), and proposing solutions to problems that will satisfy the project objectives. 


\section{underground utilities}

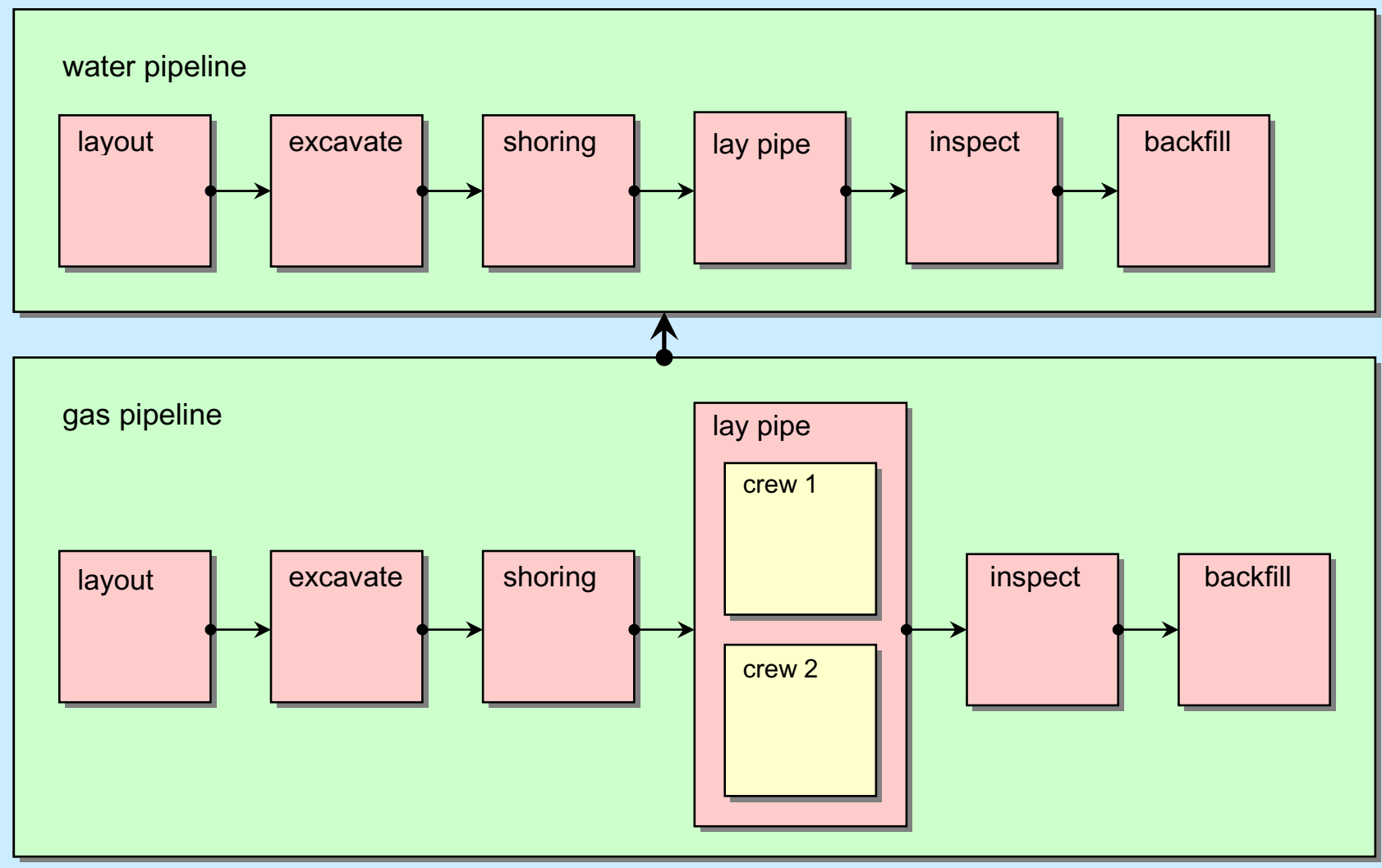

Figure 3: Basic Structure of the Model of an Underground Utilities Problem using the Proposed New Representation

While CPM activity networks and simulation diagrams are useful for understanding the work involved in a project and the dependencies between tasks, the linear scheduling diagram provides the most insight into the impact of task relationships on project progress. Linear scheduling diagrams can, incidentally, be produced as output from simulation models. CPM activity networks can (following a time analysis) be used to generate project progress curves, but these plots do not associate progress with the individual tasks, and thus provide limited visual insight into the impact of those tasks on the performance of the project.

The structured view of a project plan in the proposed approach enables visualization of progress at many levels of detail and in a format similar to that of velocity diagrams. The project task structure can be graphed to scale with, for example, time shown in one direction and some measure of progress (such as cost or activity-days) plotted in the second direction. An example of this is provided in Figure 4 for part of the output from a simulation run of the concrete production and distribution system shown in Figure 2 (with the exclusion of the intermediate wetconcrete hopper). Progress is plotted in this scaled manner within each task box (production versus time), and these task boxes can be peeled away to view progress at the higher levels in the project. This way, a user can, in an interactive environment, explore project progress at all required levels of detail. Final views of these production curves are provided in Figure 5. An advantage of this form of representation is that it is possible to see from the curves the progress of work, the idle time for individual tasks, and the points of interference between tasks, similar to the information provided by linear scheduling plots. In addition, for the intermediate level plots, it is possible to see imbalances between the tasks - the first intermediate task (concrete production) is racing ahead of the second intermediate task (concrete distribution) and then has to slow down to allow it to catch-up. This implies that one or more additional resources (distribution trucks) should be added to the concrete distribution intermediate task. 


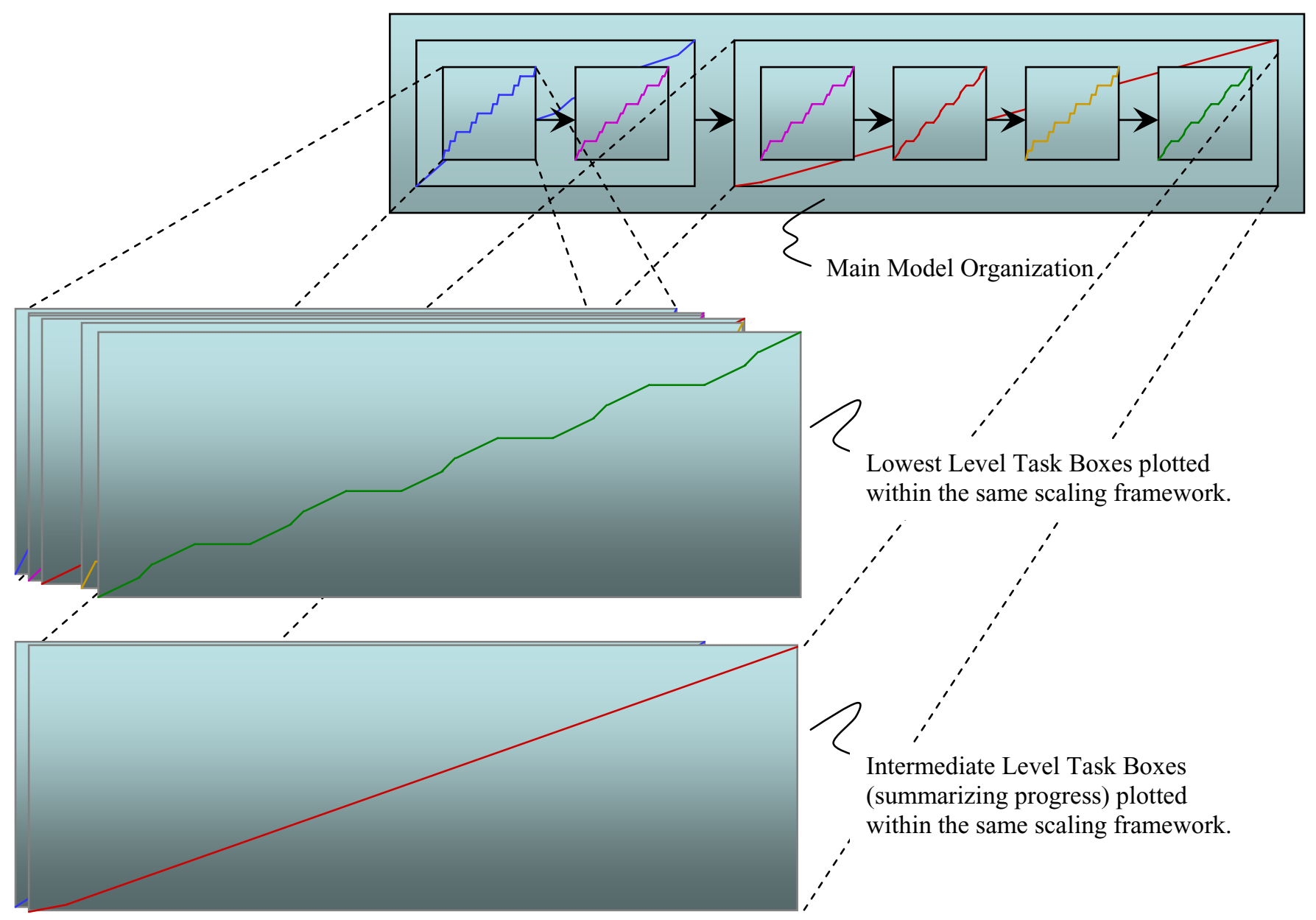

Figure 4: Concrete Foundation Pour Operation: Visualizing Progress at Different Levels of the Model

For sections of the project that are linear in nature (such as pipeline construction, tunneling, or highway construction) where several tasks follow each other on the same section of the project, the progress plots would result in something very similar to a velocity diagram. This is illustrated in Figure 6 which shows a structured model of the planned construction for the concrete structural system of a medium-rise apartment block. All boxes in the hierarchy measure 'time' in the horizontal direction. The outer level box measures 'floor level' in the vertical direction, the third level boxes measure 'square footage' in the vertical direction, and the fourth level boxes measure, for example, 'square footage' (for 'forms'), 'tons' (for 'reinforcement'), and 'cubic yards' (for 'pour concrete'). While these variables may seem incompatible and thus cannot be plotted together, the implication is that there is a linear mapping from one variable to the other, scaled to the scope of the work represented by each box. A nonlinear mapping could also be defined between two variables if the additional accuracy was considered necessary. The slight acceleration in the progress curves is the result of learning that the contractor had estimated for this pro- ject. While Figure 6 has many similarities to a velocity diagram, there are also some important differences. Most importantly, the model sits within a structured format, allowing the project to be viewed at different levels of abstraction. The different levels can be peeled away and a summary of progress at higher levels can be observed. Secondly, unlike velocity diagrams, different tasks may use different measures of progress.

\subsection{Interactive Model Development and Visualization}

An important feature of any computer-based planning tool is its ability to generate feedback on estimated project progress as each new element is added to the model. This way, the planner can see the impact of each element and each decision, on the project objectives (time, cost, etc). CPM does allow for this capability by, for example, representing progress in bar chart format, although not all implementations do it directly. In principle, linear scheduling methods can also provide this sort of information during the model development phase, although again it 
depends on the implementation. Simulation, however, is not conducive to this sort of system performance feedback during model development since generally simulation models cannot function in any meaningful way until most of the elements have been defined. A main advantage of the proposed system is that, while it has the versatility to model the same types of process as construction simulation (and indeed is implemented as a three-phase simulation algorithm) it nevertheless allows for interactive feedback on project progress during the model development stage. This is made possible in part by the structured nature of these models, which allows components to be configured in discrete units representing meaningful chunks of work.

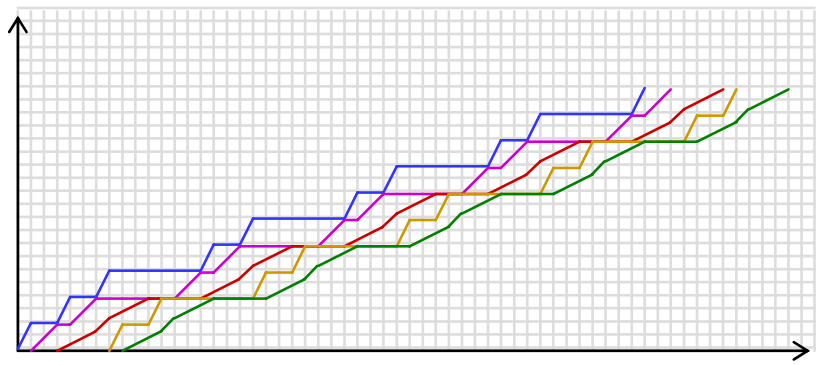

(a) Lowest Level Progress Plots

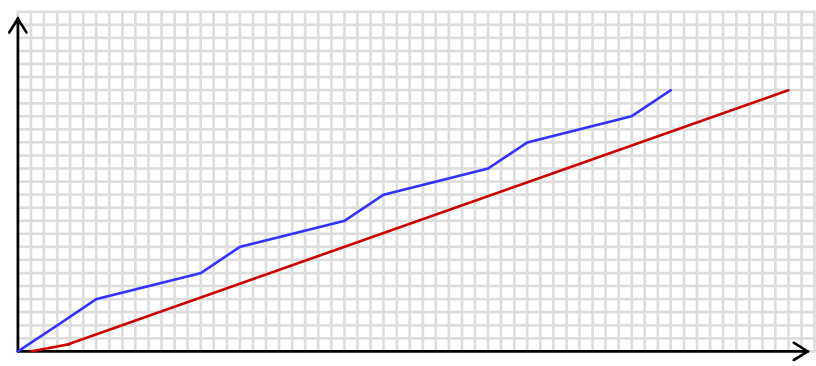

(b) Intermediate Level Progress Plots

Figure 5: Concrete Foundation Pour Operation: Final Views of Progress Plots

\section{CONCLUSIONS}

The paper has outlined a new approach to project planning and control built on principles more pertinent to contemporary project planning. It recognizes the need to facilitate planning of very large and complicated projects, achieving this by means of a truly structured representation of work, and an integration of project structure and progress within a single representation. It has the versatility to model the broad spectrum of projects that until now have required the use of several different planning methodologies.

Work is on-going developing detailed project plans using this system for a variety of project types, including underground utilities operations (water pipelines, sewers, gas pipelines, and electrical conduits) for large residential projects, high-rise condominium projects, and mediumrise office facilities. The objective of these studies is to determine the successes and limitations of the proposed planning method in the real-world, and to determine refinements that will increase its value as a planning tool.

\section{REFERENCES}

AbouRizk, S., Hajjar, D. 1998. "A Framework for Applying Simulation in the Construction Industry." Canadian Journal of Civil Engineering, 25 (3) 604-617.

Ceric, V. 1994. "Hierarchical Abilities of Diagrammatic Representations of Discrete-Event Simulation Models." Proc. of the 1994 Winter Simulation Conference, (Eds. J. D. Tew, S. Manivannan, D. A, Sadowski, and A. F. Seila) 589-594.

Flood, I., Issa, RRA., and Liu, W. 2006. “A New Modeling Paradigm for Computer-Based Construction Project Planning", Proc. of Joint International. Conference on Computing and Decision-Making in Civil and Building Engineering, Montreal, Canada, ASCE, June 2006, 11 pp.

Hajjar, D., and AbouRizk, S.M. 2002. "Unified Modeling Methodology for Construction Simulation." Journal of Construction Engineering and Management, ASCE, 128 (2), 174-185.

Halpin, D.W. and Woodhead, R.W. 1976. Design of Construction and Process Operations. John Wiley and Sons, Inc., New York, NY.

Harris, R.B., and Ioannou, P.G. 1998. "Scheduling Projects with Repeating Activities." Journal of Construction Engineering and Management. ASCE, 124 (4) 269-276.

Huber P., Jensen K. and Shapiro R.M. 1990. "Hierarchies of Coloured Petri Nets." Proc. of 10th Int. Conf. on Application and Theory of Petri Nets, (LNCS 483). Springer-Verlag. 313-341.

Issa, R.A., Flood, I., and O'Brien, W. (eds.). 2003. 4D $C A D$ and Visualization in Construction: Developments and Applications, A. A. Balkema Publishers, Steenwijk.

Koo, B., and Fischer, M., 2000. "Feasibility Study of 4D CAD in Commercial Construction." Journal of Construction Engineering and Management, ASCE, 126 (4) 251-260.

Matilla, K.G., and Araham, D.M. 1998. "LinearScheduling: past research efforts and future directions." Engineering, Construction, and Architectural Management. Blackwell Science Ltd, 5 (3) 294-303.

Sawhney, A., AbouRizk, S.M, and Halpin, D.W., 1998. "Construction Project Simulation using CYCLONE.", Canadian Journal of Civil Engineering, 25, 16-25. 


\section{AUTHOR BIOGRAPHY}

IAN FLOOD received his $\mathrm{PhD}$ from the University of Manchester, UK, in 1986, developing parallel processing methods for simulating construction operations. He has held faculty positions in the College of Architecture at the National University of Singapore, and the Department of Civil Engineering at the University of Maryland, and he is currently Holland Associate Professor at the Rinker School in the College of Design, Construction and Planning at the University of Florida. His main research focus has been the development and application of simulation and intelligent computing techniques within civil engineering. He has published over 80 refereed articles, has co-edited three books on neural networks and computerbased visualization, and is an associate editor of the ASCE Journal of Computing. 


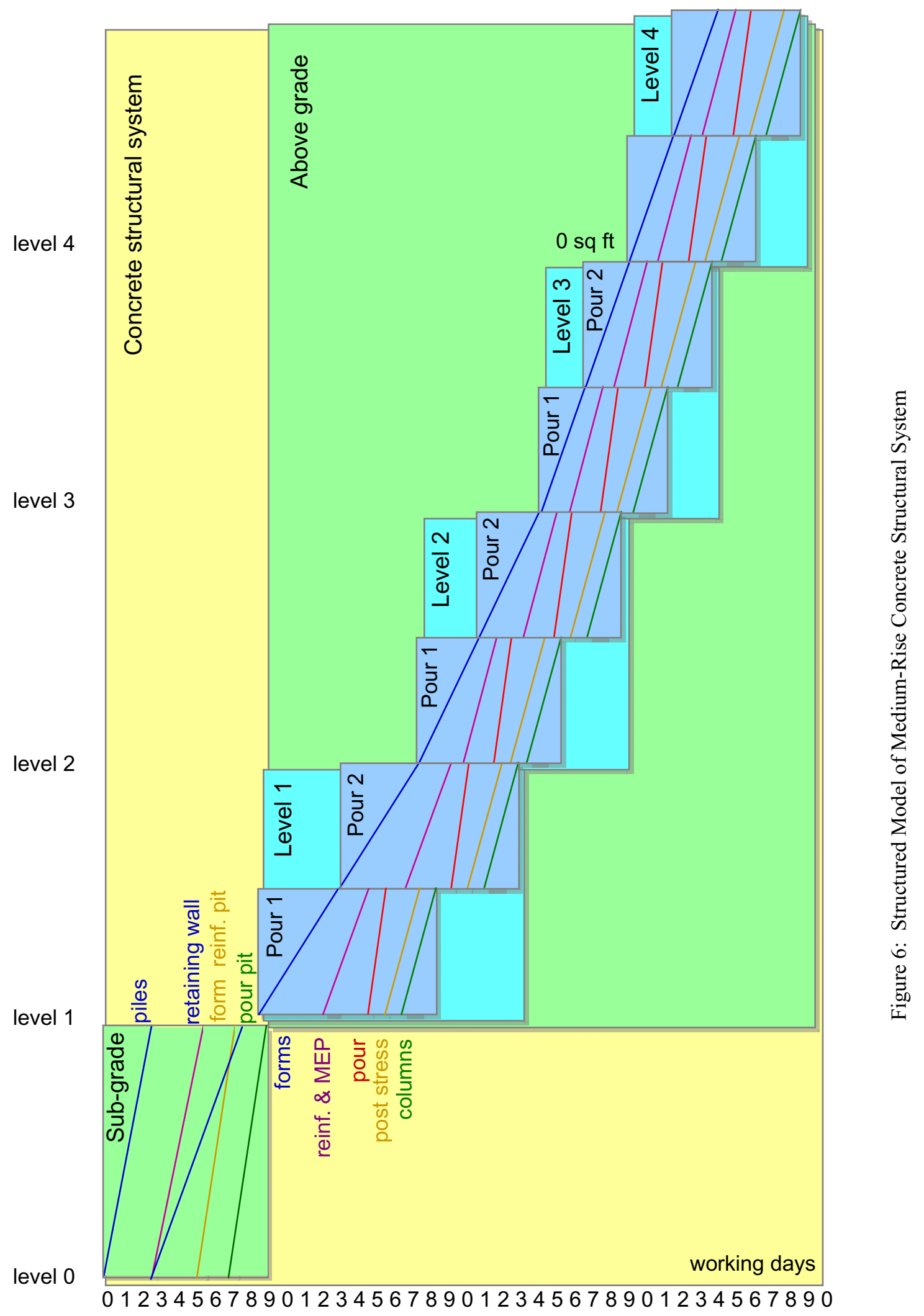

